New windows on massive stars: asteroseismology, interferometry, and spectropolarimetry

Proceedings IAU Symposium No. 30\%, 2014

G. Meynet, C. Georgy, J. H. Groh 8 Ph. Stee, eds.

(C) International Astronomical Union 2015 doi:10.1017/S174392131400742X

\title{
Interferometry of massive stars: the next step
}

\author{
Ph. Stee ${ }^{1}$ A. Meilland ${ }^{1}$ and O. L. Creevey ${ }^{2}$ \\ ${ }^{1}$ Observatoire de la Côte d'Azur - Boulevard de l'Observatoire - CS 34229 - F 06304 Nice \\ Cedex 4 - France \\ email: Philippe.Stee@oca.eu \\ ${ }^{2}$ Institut d'Astrophysique Spatiale, Université Paris XI, UMR 8617, CNRS, Batiment 121, \\ 91405 Orsay Cedex, France
}

\begin{abstract}
We present some new and interesting results on the complementarity between asteroseismology and interferometry, the detection of non-radial pulsations in massive stars and the possibility for evidencing differential rotation on the surface of Bn stars. We also discuss the curretn interferometric facilities, namely the Very Large Telescope Interferometer (VLTI)/AMBER, VLTI/MIDI, VLTI/PIONIER within the European Southern Observatory (ESO) context and the Center for High Angular Resolution Astronomy (CHARA) array with their current limitations. The forthcoming second-generation VLTI instruments GRAVITY and MATISSE are presented as well as the FRIEND prototype in the visible spectral domain and an update of the Navy Precision Optical Interferometer (NPOI). A conclusion is presented with a special emphasis on the foreseen difficulties for a third generation of interferometric instruments within the (budget limited) Extremely Large Telescope framework and the need for strong science cases to push a future visible beam combiner.
\end{abstract}

Keywords. instrumentation: interferometers, instrumentation: high angular resolution, techniques: interferometric, stars: fundamental parameters, stars: kinematics, stars: emission-line, Be, stars: winds, outflows, stars: rotation, stars: spots, stars: imaging

\section{Introduction}

I will not talk about the future of interferometry in space (Fridlund 2000b) since this is a very long term prospective and most interesting missions in space have been rejected up to now, e.g. DARWIN (Fridlund 2000a), PEGASE (Ollivier et al. 2009), SIM (Marr 2006), SIM-Lite (Goullioud et al. 2008). I will rather present the forthcoming future instruments which will be operated on the Very Large Telescope Interferometer (VLTI) and on the Center for High Angular Resolution Astronomy (CHARA) array as well as a brief overview of the current improvement of the Navy Precision Optical Interferometer (NPOI). But we first start with an example on how important interferometric radius measurements are to improve the determination of stellar masses. We will demonstrate that interferometry is really complementary to asteroseismic analysis and can help to better constrain stellar fundamental parameters.

\section{Asteroseismology \& Interferometry}

\subsection{Improving constraints on fundamental parameters.}

As already outlined by Creevey et al. (2007), interferometry and asteroseismology are providing complementary observations to determine the mass of stars. This is certainly true for solar type main sequence stars for which we have many asteroiseismic measurements. For massive stars the situation is more difficult since we don't have many 
asteroseismic constraints, which reinforce the importance of an accurate radius determination by interferometric measurements. Creevey et al. (2007) demonstrate that coupling a radius measurement of solar type stars with oscillation data improves the determination of the star's fundamental parameters while also reducing their uncertainties. While solar-like oscillations have been detected in hundreds to thousands of stars, these have been observed mostly from space and range in magnitudes from $7<V<14$. Interferometry in the visible or infrared (IR) bands, however, has a limiting magnitude of roughly $V=7$. With new instruments under development, e.g. FRIEND (Berio et al. in prep.), and adaptive optics currently being installed on the CHARA Array (ten Brummelaar et al. 2005), which hosts some of the longest baselines to observe the smallest angular diameters, the magnitude limit could be extended to $V<11$. On the other end, the recently selected ESA M3 mission PLATO (Rauer et al. 2013), with a planned launch at the end of 2024, will search for planetary transits around bright stars.

The complementarity of interferometry and asteroseismology is based on the fact that the measured p-mode oscillations and their global seismic quantities that characterise the spectrum provides a direct relation with the mean density of the star, since the measured frequency spacing $\langle\Delta \nu\rangle$ from asteroseismology is proportional to the square root of the mean density leading to Eq. 2.1.

$$
\langle\Delta \nu\rangle \simeq \sqrt{\frac{M}{R^{3}}}\langle\Delta \nu\rangle_{\odot}
$$

If the radius $R$ is measured independently and we apply Eq. 2.1 directly to determine the mass $M$ of the star, we find that for a star similar to the Sun the mass can be determined with a theoretical precision as good as $2.5 \%$ if we consider the best possible scenario $[\sigma(R)=0.5 \%$ and $\sigma(\Delta \nu)=1 \%]$, or to $5-6 \%$ for more conservative uncertainties $[\sigma(R) \sim 1-1.5 \%$ and $\sigma(\Delta \nu)=2 \%]$. This is already an improvement of at least a factor of two from using the scaling relations alone. Needless to say, the use of individual frequency separations and the frequencies themselves along with the measured radius yields uncertainties in the mass as good as 1-2\%, but again model-dependent as looked in detail below.

Creevey et al. (2007) conducted a sensitivity analysis to study the impact of an interferometric radius measurement on the determination of stellar model parameters by invoking structure and evolution models and using different types of seismic information. Asteroseismic data provide so much more information characterizing the structure of solar type stars that we are now in a position to determine these properties independent of any external assumptions. However, asteroseismic data is sensitive to the density structure of the star and thus can constrain well the determination of the relative $M$-to- $R$ relation. With $R$ measured independently, the determination of $M$ and hence the other stellar parameters can improve.

In Fig. 1 we show the predictions of the mass uncertainty (y-axis) as the precision in the radius improves (x-axis) for a star like the Sun. The different lines represent the results when different observational constraints are at hand. In all cases, spectroscopic parameters were included (effective temperature $T_{\text {eff }}$, metallicity $[\mathrm{M} / \mathrm{H}]$, surface gravity $\log g$ ) with errors of $100 \mathrm{~K}, 0.05$ dex and $0.1 \mathrm{dex}$, respectively. These values are typical of what can be found in the recent literature. The circles show the results when the asteroseismic data consist of just $\langle\Delta \nu\rangle$ and $\langle\delta \nu\rangle$, the squares using the full range of individual $l=0$ frequency separations $\Delta \nu_{l=0}$, and the diamonds when the individual $l=0$ frequencies are used. The predictions illustrate that the inclusion of the radius as a constraint is always very useful. Even including several individual oscillation modes, 


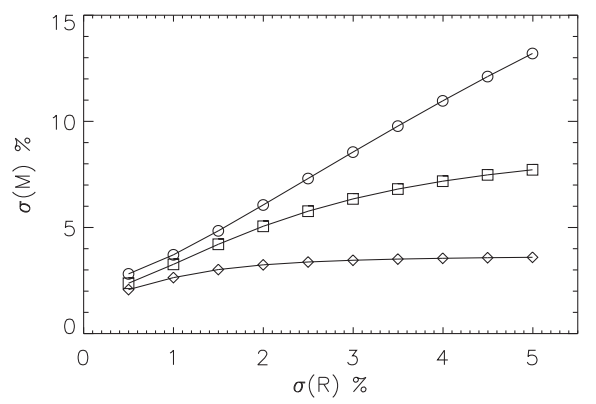

Figure 1. Predictions of the mass uncertainty (y-axis) as the precision in the measured radius improves (x-axis), when spectroscopic and asteroseismic data are also at hand. The three curves include spectroscopic constraints and the asteroseismic constraints differ from curve to curve. Circles correspond to using just the global seismic quantities, squares to including the $l=0$ large frequency separations, and diamonds to including the $l=0$ individual modes.

$<2 \%$ precision in the radius leads directly to an improvement in the mass uncertainty and in fact for a $0.5 \%$ radius error, the mass can be determined to $2 \%$.

As this sensitivity study is a linear approach that depends just on the local description of the $\chi^{2}$ surface, such results were then tested with simulations in Creevey et al. (2007). In the example that follows a slightly more massive star of $1.03 M_{\odot}$ is considered with an age of 1 billion years (one quarter that of the Sun). Using a theoretical set of observables (frequencies, $T_{\text {eff }}, .$. etc.), observational data were simulated by adding typical errors that were scaled by a random number drawn from a Gaussian distribution. These simulated data were then fitted to model data by employing a Levenberg-Marquardt minimization. The results are a set of optimal model parameters (mass $M$, age, metallicity $Z$, initial $\mathrm{H}$ mass fraction $X$, mixing-length parameter $\alpha$ ). This was repeated 50 times for each set of observations and associated errors. The observations always included the radius and spectroscopic constraints but also 1) the large frequency separations $\Delta \nu_{l, n}$ with typical errors on the individual frequencies of $0.5 \mu \mathrm{Hz}$ and and 2) the small frequency separations $\delta \nu_{l, n}$ with errors on the frequencies typical of ground-based data $(1.3 \mu \mathrm{Hz})$.

The results from the simulations are shown in Fig. 2. The continuous lines illustrate case 1 and the dashed line for the $\alpha$ parameter denotes case 2. Each point in the figure corresponds to the standard deviation of the fitted parameter for a given radius error. Four of the five parameters are shown in this figure and they are marked on the right side of the figure. Inspecting the results for $Z$ it can be seen that the radius has no impact on improving its uncertainty, it remains at a constant $\sim 10 \%$, with a similar result for $\alpha(\sim 8 \%)$. However, it is clear to see that the uncertainty in the mass does improve from an average of $3 \%$ when the seismic information dominates to $1.5 \%$ when the error in the radius reaches $0.5 \%$. This much higher precision in the mass leads directly to an improvement in the determination of $X(<2 \%)$ or, similarly, the initial He surface abundance (remember $X+Y+Z=1$ ). Including the small frequency separations, we find that the determination of $\alpha$ is also influenced by an improved radius measurement and reaches a mere $4 \%$ when this parameter is otherwise entirely unconstrained in models.

While this study highlights how important a radius measurement is to improve the determination of the stellar mass, it is of equal importance to make a direct comparison of interferometrically-determined radii with those predicted from detailed asteroseismic analysis alone. 


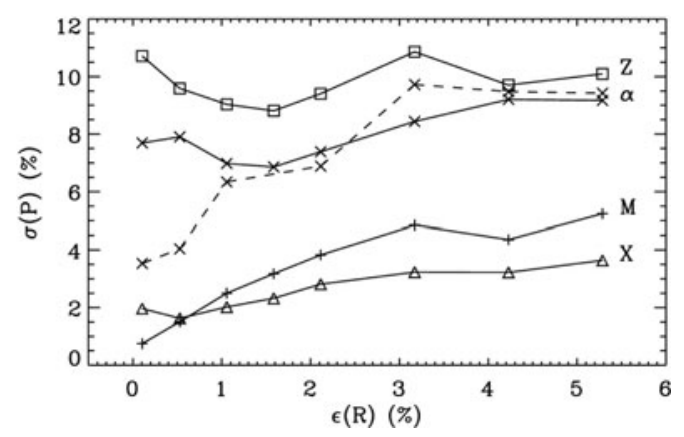

Figure 2. The uncertainties in stellar parameters (mass $M$, initial metallicity $Z$, initial hydrogen mass fraction $X$, and mixing-length parameter $\alpha$ ) as the precision in the star's measured radius improves, while considering seismic data comprising the large frequency separations (continuous) and including the small frequency separations (dashed).

\subsection{Direct detection of Non Radial Pulsations of massive stars.}

The study of the surface of rapidly rotating stars with strong non-radial oscillations using Differential Speckle Interferometry (DSI) technique was already proposed by Vakili \& Percheron (1991). The application of this technique to the study of surface structure is particularly well justified for the stars at high inclinations (Petrov 1988). Jankov et al. (2001) treated explicitly the case of non-radial stellar pulsations, for which the cancellation of opposite sign temperature or velocity fields introduces difficulties, and showed that interferometric constraint introduces the crucial improvement. In fact, the photocenter shift provides the first order moment of the spatial brightness distribution and (comparing to the zero order moment spectroscopic information) the corresponding stellar regions are reinforced by weighting with the coordinate parallel or orthogonal to rotation. Consequently, the modes that are cancelled in flux spectrum should appear in the spectrally resolved photocenter shift data. Of course, the correct detection and identification of modes present in the star is crucial for a credible asteroseismological analysis.

Following this technique, Jankov et al. (2001) propose to detect non-radial pulsations by Differential Interferometry using the dynamic spectra of photocenter shift variability characterized by bumps traveling from blue to red within the spectral lines. The theoretical estimation of expected signal-to-noise ratios in differential speckle interferometry (Chelli 1989) demonstrated the practical applicability of the technique to a wide number of sources. For instance, the high precision CHARA/VEGA or VLTI/AMBER measurements of differential fringe phase corresponding to $\sim 10 \mu$ as photocenter shift (standard limit), makes measurements feasible for the low order pulsation of $20 \mathrm{~km} \mathrm{~s}^{-1}$ and considering a star at an intermediate inclination $\left(i=45^{\circ}\right)$, which should yield a signal of about $\sim 25 \mu$ as for the typical angular diameter of $\eta$ Cen $(\sim 0.5$ mas $)$. Moreover, for $\eta$ Cen $i \sim 70^{\circ}$ and, since the star is tilted at high inclinations, the expected signals, calculated by Jankov et al. (2001), should be even stronger, particularly for low order pulsations, improving the feasibility.

\section{Measuring differential rotation on the surface of Bn stars}

Be stars are non-supergiant stars that have exhibited at least once Balmer lines in emission. These emission lines are produced in a gaseous circumstellar environment. Be stars are very rapid rotators. However, the characteristics of their rotational rate (rigid or differential) and their role in the formation of circumstellar discs are still highly debated 
(Frémat et al. 2005; Cranmer 2005). Consequently, the role of rotation in the ejection of stellar matter and the formation of the circumstellar environment from it is still an open issue (Stee \& Meilland 2009). In the past few years, significant progresses in the understanding of the structure of the circumstellar environment of Be stars were made using the new generation of spectro-interferometric instruments, mainly AMBER installed on the VLTI and VEGA installed on CHARA. For the first time, these instruments allowed to probe quantitatively the kinematics of the circumstellar gas. All the studies that were conducted (Meilland et al. 2007a; Meilland et al. 2007b; Carciofi et al. 2009; Delaa et al. 2011; Meilland et al. 2011) concluded that the disk kinematics was dominated by rotation and that expansion velocities were too small to be detected (i.e. of the order of $1 \mathrm{~km} \mathrm{~s}^{-1}$ ). Finally, the first statistical study of the disks kinematics by Meilland et al. (2012) showed that the rotation law within the disk was nearly Keplerian and that the inner boundary of the disk (i.e. near the stellar surface) was rotating very close to the stellar critical velocity, with an angular rotation velocity relative to critical of $\frac{\Omega}{\Omega_{\mathrm{c}}} \sim 0.95$.

However, even though $\frac{\Omega}{\Omega_{c}}$ can be very high it still underestimates the actual stellar rotation, which is more clearly evidenced by the $\eta$ parameter. $\eta$ is defined as the ratio of the centrifugal and the gravitational forces at the stellar equator. Thus, the measurement of $\eta$ is crucial if we would like to know how close are Be stars to critical rotation. Unfortunately, Be stars can have photospheric spectral lines marred by emission/absorptions due to their circumstellar disc. Bn stars, which are nearly as fast rotators as Be stars, don't have spectra perturbed by circumstellar matter, so that the study of their apparent geometry can be carried out more properly and reliably.

Due to the rapid rotation, the surface geometry of Bn and Be stars is highly deformed. The surface angular velocity can be dependent on the stellar latitude. Then, not only the centrifugal force acting on the stellar surface, but also the effective temperature distribution (Von Zeipel effect) should depend on the surface rotation law. Up to now, these effects have been measured only for few stars (Domiciano de Souza et al. 2003; Monnier et al. 2007; Zhao et al. 2009; Che et al. 2011) and different values of the $\beta$ exponent of the gravity darkening law were deduced using image reconstruction techniques(Monnier et al. 2007; Zhao et al. 2009; Che et al. 2011). Delaa et al. (2013) has demonstrated that a differential rotation at the surface of fast rotating star may affect the brightness distribution of the stellar disc and, so, also modify the value of the $\beta$ exponent. Consequently, a value of $\beta$ different from 0.25 (for a fully a radiative envelope; von Zeipel 1924) can also be due to a non-conservative rotational law in the outermost stellar layers (Zorec et al. 2011) and can be constrained by spectrally resolved interferometric measurements. The latitude dependent angular velocity also introduces peculiar characteristics to the spectral line differential phases. These last are sensitive to the stellar inclination angle and to the surface angular velocity law (Delaa et al. 2013).

\section{The Very Large Telescope Interferometer (VLTI)}

The VLTI, located on Paranal (Chile), is equipped with two general-user focal instruments: the Astronomical Multi-BEam combineR (AMBER; Petrov et al. 2007; RobbeDubois et al. 2007) and the MID-infrared Interferometric instrument (MIDI; Leinert et al. 2003) beam combiners. MIDI can combine only 2 telescopes in the $8-13 \mu \mathrm{m}$ spectral bandwidth with resolving power $\mathrm{R}=30$ and 230 , and can measure visibilities and differential phases. The spatial resolution is about 10 mas. AMBER operates in the nearIR bands $(J, H$,and $K)$ and can combine 3 telescopes simultaneously with 3 spectral 
resolution modes: $\mathrm{R}=30,1500$ and 12000 . It can measure visibilities, differential phases and phase closure. Its spatial resolution is about 2 mas.

\subsection{Current strengths and limitations}

The VLTI is currently the best angular resolution facility accessible in Europe. Equipped with $8 \mathrm{~m}$ class telescopes (UT), the magnitude limit in the $\mathrm{K}$ band $(2.2 \mu \mathrm{m})$ is now $\sim 11$ (Petrov et al. 2012). After a huge investment, it is now working well and the $1.8 \mathrm{~m}$ Auxiliary Telescopes (AT) array is very powerful. It is operated within the ESO framework and thus benefits from various facilities such as service mode observations, archives, maintenance and good stability. This instrument is very powerful for the study of multiplicity (e.g., massive stars, brown dwarfs, YSOs), temporal variability of various objects (e.g., YSOs, Novae, evolved stars, MIRAS, Be stars) and benefits from a very active and well organized community (e.g., Schools, European Interferometric Initiative, Jean-Marie Mariotti Center - JMMC). Two new instruments are coming up (MATISSE and GRAVITY) and the VLTI offers a potential synergy with ALMA.

On the other hand, the community is small and partly "interferocentric". The accessibility to reduced data is still a problem 10 years after the start of operations. Longbaselines are missing, which is a strong limitation for resolving most of the stellar surfaces (a typical diameter for a B-type star is about 0.5 mas within our Galaxy). The funding context within the Extremely Large Telescope (ELT) era is hostile and the ESO current organization context (no more Paranal VLTI group, probably no more Garching VLTI group) weakens the possibility to develop the VLTI in a holistic way. Regarding the present focal instruments, MIDI can only estimates sizes of objects (no real information on the geometry with only two telescopes). It has no imaging capabilities nor (or few) gas study possibilities. AMBER has a limited accuracy and limited imaging capabilities and dust analysis are limited to (very) hot dust. In addition, the $K$ - and $N$-bands have been used a lot and we need to open new wavelength windows. We also have to notice that with 2 telescopes we can measure only two quantities ( 1 visibility +1 differential phase), with 3 telescopes we have access to 7 measurements ( 3 visibilities, 3 differential phases, 1 closure phase), with 4 telescopes we have 16 measurements accessible (6 visibilities, 6 differential phases, 3 closure phases and 1 closure amplitude) and thus going from 3 to 4 telescopes more than doubles the number of available measurements. This is currently the success of the new visiting Precision Integrated-Optics Near-Infrared Imaging ExpeRiment (PIONIER) instrument which can combine the light from 4 telescopes simultaneously in the near-infrared $(1.5-2.4 \mu \mathrm{m})$ domain (Le Bouquin et al. 2011).

\subsection{The forthcoming VLTI instruments GRAVITY and MATISSE}

GRAVITY is a four-way beam combination, second generation instrument for the VLTI. Its main operation mode makes use of all four $8 \mathrm{~m}$ Unit Telescopes to measure astrometric distances between objects located within the 2" field-of-view of the VLTI (Eisenhauer et al. 2011). With the sensitivity of the UTs $(8 \mathrm{~m})$ and the $\sim 10 \mu$ as astrometric precision (for a $5 \mathrm{mn}$ integration), it will allow to measure orbital motions within the galactic center with unprecedented precision. GRAVITY is dedicated to the study of the back hole at the center of our Galaxy and will carry out the ultimate empirical test to show whether or not the Galactic Centre harbors a black hole of four million solar masses. It will also provide a high-precision narrow-angle astrometry and phase-referenced interferometric imaging in the astronomical K-band. The resolving power will be $R=$ 22 , 500, and 4000, which will be very interesting for the study of circumstellar disks around massive stars and interacting binaries or Be-X ray sources. Other modes of the instrument will allow imaging and the use of the ATs. It will allow fringe tracking up to 
$\mathrm{K} \sim 10$ with the UTs and $\mathrm{K} \sim 7$ with the ATs. It will be able to produce images up to $\mathrm{K} \sim 16$ (UTs) and $\mathrm{K} \sim 13$ (ATs) for a $100 \mathrm{~s}$ integration. It will start operations in mid-2015.

The Multi AperTure mid-Infrared SpectroScopic Experiment (MATISSE) is a midinfrared $(L-, M-$ and $N$-bands; i.e. $3.7-5 \mu \mathrm{m}$ and $8-13 \mu \mathrm{m})$ spectro-interferometer project combining up to four UTs/ATs beams of the VLTI. It will measure closure phase relations, thus offering an efficient capability for image reconstruction. This mid-infrared instrument will include several efficient spectroscopic modes ranging from $R=20$ to 1500. The typical spatial resolution will be $5-40$ mas for baselines ranging from 8 to $150 \mathrm{~m}$. MATISSE is supposed to be operational just after GRAVITY in 2016.

\section{Update of other interferometric arrays}

\subsection{The CHARA array}

CHARA is located at Mount Wilson Observatory, California and is an optical interferometric array of six $1 \mathrm{~m}$ telescopes. The longest available baseline of $330 \mathrm{~m}$ makes the CHARA array the highest spatial resolution instrument in the world. Actually, 7 beam combiners are operational:

- CHARA Classic: 2 telescopes open air beam combiner in J, H and $\mathrm{K}$ band, limited magnitude of 6.5 in $\mathrm{K}$.

- CHARA CLIMB: 3 telescopes open air in J,H,K

- JouFLU (upgrade of FLUOR: 2 telescopes, fiber based in K band, limited magnitude of $4-5$ in $\mathrm{K}$

- MIRC: 6 telescopes fiber based imager in $\mathrm{H}$ and $\mathrm{K}$, spectral resolution R: 35, 150, 450, limited magnitude of 4-5 in $\mathrm{H}$

- VEGA: 4 telescopes, open air in V, R and I with a spectral resolution up to 30000, limited magnitude of 7 in $\mathrm{V}$

- PAVO: 3 telescopes, aperture plane in $\mathrm{V}, \mathrm{R}$ and $\mathrm{I}$ bands, limited magnitude of 8 in $\mathrm{V}$

- CHAMP: 6 telescopes pair wise fringe tracker in $\mathrm{H}$ and $\mathrm{K}$

At the Observatoire de la Côte d'Azur we are trying to improve the VEGA beam combiner limitations, such as mainly no closure phase possible, difficulties to measure low visibilities and limitations of the measurement's accuracy due to the multimode regime, the saturation effect of the photon counting detector and the photon centroiding "hole" (Mourard et al. 2009; Mourard et al. 2011). We are developing a prototype named "FRIEND" for Fibered and spectrally Resolved Interferometric Experiment - New Design (Berio et al. 2014, in preparation) that can benefit from the forthcoming adaptive optics on the CHARA array. The FRIEND prototype will combine 3 telescopes with spectrally dispersed fringes, i.e. $\mathrm{R}=1500$ (as VEGA and AMBER) in the $\mathrm{R}$ band $(0.6-0.8 \mu \mathrm{m})$. It will use spatial filtering thanks to fibers (as MIRC and AMBER), a V-groove (as MIRC) and simultaneous photometry (as AMBER). Finally the fringes will be sampled thanks to a low noise $(<\mathrm{rev})$ visible detector OCAM2. The predicted performances will be as good as $\mathrm{m}_{v} \sim 10$ with the full CHARA adaptive optics system. First tests of FRIEND at CHARA are foreseen mid december 2014 with tip-tilt only. The long term perspective of FRIEND is to combine up to 6 telescopes (from the VLTI or CHARA) in the visible with $300 \mathrm{~m}$ baselines which will enable stellar surface imaging of massive stars. 


\subsection{The NPOI interferometer}

NPOI is a six $12.5 \mathrm{~cm}$ apertures array on a $\mathrm{Y}$ shape with baselines up to $79 \mathrm{~m}$ located near Flagstaff, Arizona (Armstrong et al. 1998). It has been recently upgraded with the completion of 6 stations "imaging" (portable) siderostat array: new enclosures for star acquisition and tip-tilt optics have been installed for 5 of 6 stations. The news domes are installed for 5 of 6 imaging siderostats and 2 more imaging stations will be commissioned in 2014. A long baseline up to $432 \mathrm{~m}$ and a "compact" configuration will be available. The "classic" combiner operates at low spectral resolution (16 spectral channels from $550 \mathrm{~nm}$ to $850 \mathrm{~nm}$ ) with a limiting magnitude of $\mathrm{m}_{v} \sim 6$. The second beam combiner VISION uses single-mode polarization-preserving fibers to spatially filter the incoming beams and a EMCCD (Electron Multiplying Charge Coupled Device). It allows a full 6 -way combination with flexible spectral resolution $(R=500,2000)$ and a visibility measurement precision 10 times better than the "classic" beam combiner. Last but not least, they will replace the $12.5 \mathrm{~cm}$ siderostats by $1.8 \mathrm{~m}$ telescopes that have been built by NASA for the Keck interferometer. The site install will start in October 2014.

\section{Conclusion}

With the forthcoming second generation of VLTI instruments, the CHARA array equipped with adaptive optics and the promising NPOI interferometer, the massive star research will benefit from new imaging facilities, good spectral coverage and very high spatial resolution that will enable the imaging of massive star surfaces in our Galaxy. Nevertheless, within the ELT framework and the limited budget allocated to future projects, the third generation of interferometers will be very competitive. For instance, due to the limitation of the Paranal mountain, no baselines larger than $\sim 200 \mathrm{~m}$ are foreseen. Thus a possible way to increase the spatial resolution without increasing the baseline is to observe at smaller wavelengths. I.e. going from the near-IR to visible will increase the spatial resolution by a factor 4 ! On the other side, third generation instruments will only be possible if we are able to extend the community to non-interferometry people and convince ESO or other institutions that we have very strong science cases for a visible instrument. Thus, if you are interested, don't hesitate to join us and contact our group for future collaborations.

\section{References}

Armstrong, J. T., Mozurkewich, D., Rickard, L. J., et al. 1998, ApJ 496, 550

Carciofi, A. C., Okazaki, A. T., Le Bouquin, J.-B., et al. 2009, A\& A 504, 915

Che, X., Monnier, J. D., Zhao, M., et al. 2011, ApJ 732, 68

Chelli, A. 1989, AESA 225, 277

Cranmer, S. R. 2005, ApJ 634, 585

Creevey, O. L., Monteiro, M. J. P. F. G., Metcalfe, T. S., et al. 2007, ApJ 659, 616

Delaa, O., Stee, P., Meilland, A., et al. 2011, A $6 A$ 529, A87

Delaa, O., Zorec, J., Domiciano de Souza, A., et al. 2013, A 6 A 555, A100

Domiciano de Souza, A., Kervella, P., Jankov, S., et al. 2003, A\&A 407, L47

Eisenhauer, F., Perrin, G., Brandner, W., et al. 2011, The Messenger 143, 16

Frémat, Y., Zorec, J., Hubert, A.-M., \& Floquet, M. 2005, A\&A 440, 305

Fridlund, C. V. M. 2000a, in B. Schürmann (ed.), Darwin and Astronomy : the Infrared Space Interferometer, Vol. 451 of ESA Special Publication, p. 11

Fridlund, C. V. M. 2000b, in P. Léna \& A. Quirrenbach (eds.), Interferometry in Optical Astronomy, Vol. 4006 of Society of Photo-Optical Instrumentation Engineers (SPIE) Conference Series, pp 762-771 
Goullioud, R., Catanzarite, J. H., Dekens, F. G., Shao, M., \& Marr, IV, J. C. 2008, in Society of Photo-Optical Instrumentation Engineers (SPIE) Conference Series, Vol. 7013 of Society of Photo-Optical Instrumentation Engineers (SPIE) Conference Series

Jankov, S., Vakili, F., Domiciano de Souza, Jr., A., \& Janot-Pacheco, E. 2001, A\&A 377, 721

Le Bouquin, J.-B., Berger, J.-P., Lazareff, B., et al. 2011, A\& A 535, A67

Leinert, C., Graser, U., Przygodda, F., et al. 2003, Ap\&SS 286, 73

Ligi, R., Mourard, D., Lagrange, A. M., et al. 2012, A\& A 545, A5

Marr, IV, J. C. 2006, in Society of Photo-Optical Instrumentation Engineers (SPIE) Conference Series, Vol. 6268 of Society of Photo-Optical Instrumentation Engineers (SPIE) Conference Series

Meilland, A., Delaa, O., Stee, P., et al. 2011, A\& $A$ 532, A80

Meilland, A., Millour, F., Kanaan, S., et al. 2012, A\&A 538, A110

Meilland, A., Millour, F., Stee, P., et al. 2007a, A\&A 464, 73

Meilland, A., Stee, P., Vannier, M., et al. 2007b, A\&SA 464, 59

Monnier, J. D., Zhao, M., Pedretti, E., et al. 2007, Science 317, 342

Mourard, D., Bério, P., Perraut, K., et al. 2011, A\&S 531, A110

Mourard, D., Clausse, J. M., Marcotto, A., et al. 2009, A\&\&A 508, 1073

Ollivier, M., Absil, O., Allard, F., et al. 2009, Experimental Astronomy 23, 403

Petrov, R. G. 1988, in D. M. Alloin \& J.-M. Mariotti (eds.), Diffraction-Limit.Imaging/Very Large Telescopes, p. 249

Petrov, R. G., Malbet, F., Weigelt, G., et al. 2007, A\& $A$ 464, 1

Petrov, R. G., Millour, F., Lagarde, S., et al. 2012, in Society of Photo-Optical Instrumentation Engineers (SPIE) Conference Series, Vol. 8445 of Society of Photo-Optical Instrumentation Engineers (SPIE) Conference Series

Rauer, H., Catala, C., Aerts, C., et al. 2013, ArXiv e-prints

Robbe-Dubois, S., Lagarde, S., Petrov, R. G., et al. 2007, A\&A 464, 13

Stee, P., Delaa, O., Monnier, J. D., et al. 2012, A $\& A$ 545, A59

Stee, P. \& Meilland, A. 2009, in J.-P. Rozelot \& C. Neiner (eds.), The Rotation of Sun and Stars, Vol. 765 of Lecture Notes in Physics, Berlin Springer Verlag, pp 195-205

ten Brummelaar, T. A., McAlister, H. A., Ridgway, S. T., et al. 2005, ApJ 628, 453

Vakili, F. \& Percheron, I. 1991, in D. Baade (ed.), European Southern Observatory Conference and Workshop Proceedings, Vol. 36 of European Southern Observatory Conference and Workshop Proceedings, p. 77

von Zeipel, H. 1924, MNRAS 84, 665

Zhao, M., Monnier, J. D., Pedretti, E., et al. 2009, ApJ 701, 209

Zorec, J., Frémat, Y., Domiciano de Souza, A., et al. 2011, A\& A 526, A87

\section{Discussion}

WADE: When will we get circularly polarized spectro-photometric interferometry?

STEE: It is not planned for the second generation VLTI instruments and the foreseen projects. But you certainly know that we have already implemented a polarimeter within the VEGA focal instrument on the CHARA array composed of a Wollaston prism to separate two orthogonal polarization states and a movable quarter wave plate. A fixed quarter wave plate is placed after the Wollaston prism to transform the two linearly polarized output beams into two circularly polarized beams. See Mourard et al. (2009) and Stee et al. (2012) for more details.

AERTS: Could you elaborate on the dependence of the data and the calibrators on the assumptions on the limb darkening models and how that propagates into an uncertainty on the stellar radius?

STEE: Calibrating interferometric measurements is a science in itself and finding a good calibrator is not an easy task. Fortunately the JMMC provide a tool named "SearchCal" 
to assist the astronomers in this calibrator selection process for long baseline interferometric observations. To constrain the limb darkening you need first to access to second and third visibility "lobes", i.e. access to very faint and small visibility amplitudes $(\sim 2-3 \%)$. It means a good photometric calibration is needed in order to reach a precision of a few percent in the limb-darkened diameter. This is clearly something that is better suited for fiber-based interferometers with well-controlled photometry as the FLUOR or MIRC beam combiners. In order to constrain the limb-darkened diameter, people have often used the Claret \& Bloemen tables but they have to take care because the limb darkening is also wavelength dependent and thus there is not only "one" limb-darkened radius. Of course, even if you have very accurate interferometric measurements, you also need a very accurate calibrator diameter, otherwise your final uncertainty will be due to the error on the calibrator diameter. This is clearly the case when you are calibrating data in the visible with very large baselines (larger than $200 \mathrm{~m}$ ) where it is very difficult to find unresolved calibrators and very often your calibrator is more resolved than your science star! Fortunately, the forthcoming GAIA measurements will help to better constrain the stellar distances and thus the precisions on the calibrator's diameters. A nice work and a discussion about interferometric limb-darkened diameters of exoplanet host stars can be found in Ligi et al. (2012).

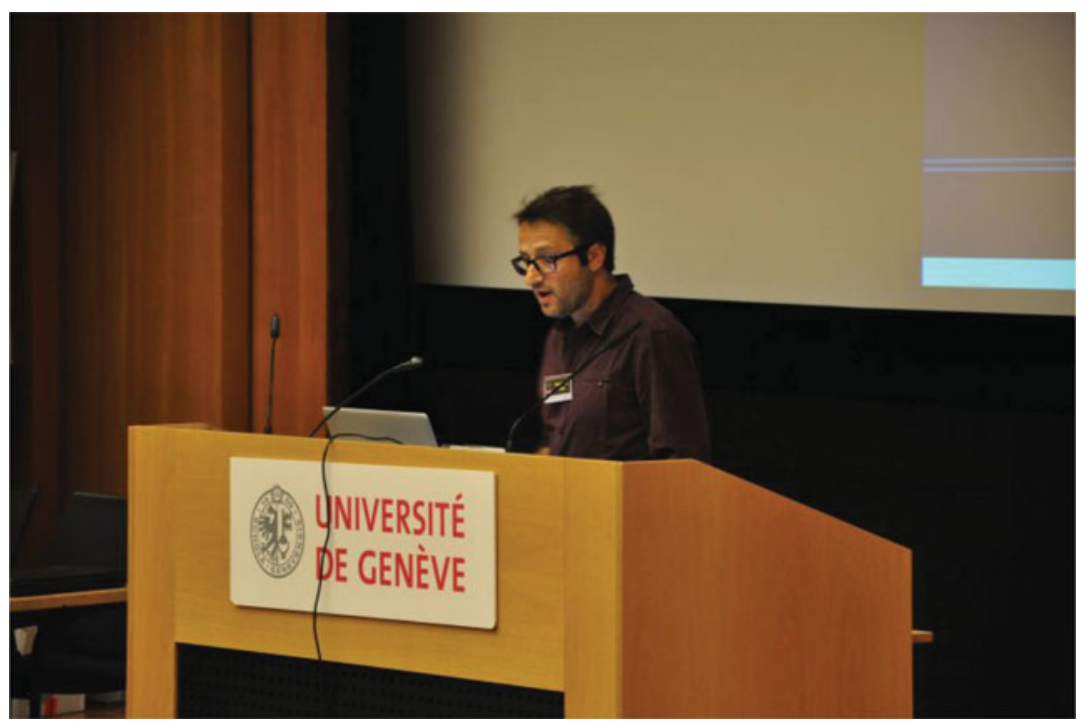

Philippe Stee 